\title{
62-TEV CENTER OF MASS HADRON COLLIDER WITH CAPABILITY FOR SUPER BUNCH BEAMS
}

\author{
R. Yamada*, FNAL, Batavia, IL 60510, USA \\ K. Takayama, J. Kishiro, M. Wake, T. Toyama, Y. Shimosaki, KEK, Tsukuba, 305-0801, Japan
}

\begin{abstract}
A two staged $62 \mathrm{TeV}$ center of mass hadron collider is proposed. In the first stage the center of mass energy will be $14 \mathrm{TeV}$ with 2 Tesla dipole magnets. With Superbunch beams, the luminosity is expected to be increased by a factor of 15 , compared with conventional acceleration using RF cavities. To accelerate Superbunch beams, the barrier bucket induction cells and acceleration induction cells will be used, which are made of FINEMET material. The core loss of the FINEMET is estimated to be acceptable. The synchrotron radiation of the collider is also estimated. In the second stage, 10 Tesla high field dipole magnets will be installed, and the application of Superbunch is being studied.
\end{abstract}

\section{INTRODUCTION}

As a future project in US high energy physics, the VLHC has been proposed for the future hadron collider $[1,2]$. As a variant scheme a two-staged 25-30 TeV P-P collider was proposed [3]. The LHC, 14-TeV Center of mass hadron collider [4] is being constructed now at CERN, and probably will be in operation in 2006. We should design and build a hadron collider in US, which eventually surpass the luminosity and energy of the LHC, but it should be conceived as a realizable machine, budgetwise, spacewise, and timewise. The machine should be designed also as easily maintainable with present-day's technology.

Recently proposals of applying induction acceleration to synchrotrons and hadron colliders were made, with the increase of the luminosity about 15 times as their advantage over the RF cavity acceleration $[5,6,7]$. We apply this induction acceleration to the design of the 62 $\mathrm{TeV}$ center of mass hadron collider with Superbunch beams in both stages.

\section{GENERAL DESCRIPTIONS}

This collider complex will be based at Fermilab site, and built in two stages, utilizing a same tunnel. The Fermi Main Injector of $150 \mathrm{GeV}$ proton beams will be used as the injector to the first stage. In the first stage a low field magnet ring made of 2 Tesla superferric magnets will be installed [1]. The beams will be accelerated to $7 \mathrm{TeV}$ with accelerating induction cells and barrier bucket induction cells to produce Superbunch beams $[5,6]$. The maximum center of mass colliding energy will be $14 \mathrm{TeV}$ with the

*yamada@fnal.gov expected luminosity of $1.5 \times 10^{35} / \mathrm{cm}^{2} / \mathrm{sec}$. This will be used for high energy experiments with two detectors.

In the second stage we will use the twin-aperture 10 Tesla superconducting magnets. The operational dipole magnetic field of 10 Tesla using $\mathrm{Nb}_{3} \mathrm{Sn}$ conductor, is achievable with the present technology. The maximum accelerated proton energy will be $31 \mathrm{TeV}$, resulting in the center of mass energy of $62 \mathrm{TeV}$. In this second stage, the application of Superbunch beams is being studied to get the expected luminosity of $5 \times 10^{34} / \mathrm{cm}^{2} / \mathrm{sec}$ without generating too much synchrotron radiation loss. The basic parameters of this hadron colliders complex are given in Table 1.

\section{TUNNEL}

The tunnel will be placed at a depth of 130 meters from the surface. The $62 \mathrm{TeV}$ collider has the total circumference of 87.25 kilometers. The layout of the 87.25 kilometer tunnel is shown in Fig.1. Inside the Fermilab site there will be two major $1 \mathrm{~km}$ long straight sections, where two major $62 \mathrm{TeV}$ hadron collider detectors will be installed. These two detectors will be used for the collider experiments with the $14 \mathrm{TeV}$ center of mass energy in the first stage. In the second stage, the low field ring will be lifted up by 2 meters to make room for the high field magnet ring, keeping the two major detectors at the same position. Also the part of the low field ring inside Fermilab site will be moved into an outward bypass.

In addition to two long straight sections, there will be three 300 meter utility straight sections in the Fermilab site. Two of them will be used for the injection from the Main Injector to the low field magnet ring. They will be used also for transferring two proton beams from the low field magnet ring to the high field magnet ring. The induction cells and the liquid Helium connection will be placed in the central 300 meter long straight section. The abort lines will start from the injection straight sections, and share partly the outward bypass lines. On the other side of the tunnel, opposite to Fermilab site, there will be two $1 \mathrm{~km}$ long straight sections and three short 300 meter utility straight sections for future usage.

The cross section of the proposed tunnel for the $62-\mathrm{TeV}$ collider is shown in Figure 2. The high field superconducting magnet for the $62-\mathrm{TeV}$ collider is shown together with the magnet of the $14-\mathrm{TeV}$ collider ring. A cryogenic pipeline for transporting liquid helium is also shown. For reasonable maintenance of the collider components, the radius of the tunnel is chosen to be 3 
meters. It is imperative to have a fast transit system to be installed inside the tunnel for the initial installation of components and for transportation of personnel as well as for moving parts for maintenance.

\section{INDUCTION CELL ACCELERATION SYSTEM}

With the induction cell acceleration, the acceleration and longitudinal focusing are independently achieved. It allows ultimate use of longitudinal phase-space and is quite effective to substantially increase the beam intensity in synchrotrons [5]. We need two types of induction cells for this system, one for the accelerating induction cells, called A-IC, and the other for the barrier bucket induction cells, called BB-IC. These induction cells are now being developed at KEK [7].

\subsection{Design and Coreloss of Acceleration Induction Cell}

A set of four FINEMET FT-3M cores, each with dimensions of $\mathrm{ID}=10 \mathrm{~cm}, \mathrm{OD}=50 \mathrm{~cm}$ and width $=1 \mathrm{~cm}$, is used to generate a $2.5 \mathrm{kV}$ pulse with $0.45 \mu$ s pulse width. Two sets of cores will be used as a unit induction cell for the first stage, and only one set for the second stage.

The coreloss for unit induction cells are $4 \mathrm{~kW}$ and $0.45 \mathrm{~kW}$, and the maximum total peak coreloss will be 1.7 and $0.52 \mathrm{MW}$ for the first and second stages respectively, including synchrotron radiation effect. The cooling of cores is a challenging job, but can be done.

\section{SUPERBUNCH PRODUCTION}

With the circumference of $87.25 \mathrm{~km}$, it takes 291 microseconds to go around the ring. In the first stage we can divide the circumference into 114 units, 765 meter long each. The bunch spacing is $2.55 \mu \mathrm{sec}$. In one induction unit cell there are two sets of cores, each set of cores generating a $0.45 \mu \mathrm{sec}$ pulse in succession, making a $0.9 \mu \mathrm{sec}$ composite long acceleration pulse. At the both ends of this pulse, there are barrier bucket pulses. And in the following $1.5 \mu \mathrm{sec}$, both sets of cores are reset. These cores are triggered by high current semiconductor switches, which can be operated precisely and reliably.

\section{INJECTION INTO 7 AND 31 TEV RINGS}

The $150 \mathrm{GeV}$ Main Injector will provide proton beams to both bores of the $7 \mathrm{TeV}$ Low Field Magnet Ring, as shown in Fig.1. These two proton beams are accelerated to $7 \mathrm{TeV}$ simultaneously. Later in the second stage, these $7 \mathrm{TeV}$ beams are injected into the two bores of the High Field Magnet Ring, and accelerated to $31-\mathrm{TeV}$ beams simultaneously.

\section{BEAM PHYSICS ISSUES AND LUMINOSITIES}

In the Superbunch scheme the continuous parasitic collision is a big issue. Certainly the tune shift for particles within $1 \sigma$ is remarkably suppressed by utilizing the hybrid crossing, as explained in a previous paper [6]. To suppress the amplitudes of large amplitude particles, the crossing angle should be increased to $400 \mu \mathrm{rad}$. at the expense of $50 \%$ loss in the luminosity. Even with this, a $14 \mathrm{TeV}$ center of mass Superbunch hadron collider can deliver the luminosity of $1.5 \times 10^{35} / \mathrm{cm}^{2} / \mathrm{s}$. Since the 62 $\mathrm{TeV}$ center of mass Superbunch hadron collider has the same situation, we could expect the luminosity over $1 \mathrm{x}$ $10^{35} / \mathrm{cm}^{2} / \mathrm{s}$. But due to the big synchrotron radiation loss we should like to limit the luminosity in the order of $5 \mathrm{x}$ $10^{34} / \mathrm{cm}^{2} / \mathrm{s}$.

In the Superbunch scheme the bunch length is extremely long, about 150 to $300 \mathrm{~m}$, compared with that in the RF bunch scheme. However the bunch is not continuous because it is confined in a barrier bucket with a small synchrotron oscillation frequency. The growth time in the longitudinal direction due to intrabeam scattering is negligible, while that in the transverse direction it is of similar order as the growth time in the RF bunch scheme.

\section{EFFECTS OF SYNCHROTRON RADIATION AND BEAM SCREEN}

In the first stage, the synchrotron radiation loss is 0.18 $\mathrm{W} / \mathrm{m} /$ beam with full beam intensity as designed. And as it is in the vacuum tube at room temperature, there is no serious problem.

In the second stage, we have to install a beam screen inside the vacuum tube, because of synchrotron radiation of the beam. The beam screen has a very complicated and delicate mechanical structure that also makes the vacuum problem a complex subject to be studied carefully [1].

The energy loss per meter of the synchrotron radiation scales as $\mathrm{E}^{2} \times \mathrm{B}^{2} \times \mathrm{I}$, where $\mathrm{I}$ is the beam current. For the 62-TeV CM P-P collider the estimated energy loss due to the synchrotron radiation is $89 \mathrm{~W} / \mathrm{m} /$ beam, if we consider the extension from the first stage. Therefore we should like to limit the average current, by reducing the number of Superbunches. By selecting the number of Superbunches to be 26 , the Synchrotron radiation can be cut to $12 \mathrm{~W} / \mathrm{m} /$ beam, and the luminosity can be $5 \times 10^{34}$ $/ \mathrm{cm}^{2} / \mathrm{s}$. We still have to optimize the situation.

\section{CONCLUSIONS}

As a scenario, we should start building the 7-TeV first stage ring as soon as possible. At the same time we should start designing and building the collider detectors, which will be used first as the $14-\mathrm{TeV}$ CM collider detector, but with the intention to expand them into $62-\mathrm{TeV} \mathrm{CM}$ collider detectors. After several years of experiments at $14 \mathrm{TeV}$ CM energy and whenever the high field magnets are ready to be installed, we should switch to the $62-\mathrm{TeV}$ $\mathrm{CM}$ collider. In this way, we can keep the high energy physics experiments running and high energy physicists working in the USA.

The 62-TeV P-P collider should be started with a lower luminosity. Eventually we should get the luminosity of 
$1 \times 10^{35} / \mathrm{cm}^{2} / \mathrm{sec}$. The only components to be developed for this collider are the long 10-T high field superconducting magnets with good beam screen.

The induction cells can be built with the present industrial material, but we hope the industry will make material with less core loss in the near future.

\section{REFERENCES}

[1] "Design Study for a Staged Very Large Hadron Collider," TM-2149, Fermilab internal report, June 4, 2001.

[2] C.S. Mishra and P.J. Limon "Design Parameters for the Very Large Hadron Collider," Proceedings of the 1999 Particle Accelerator Conference, 2644-2646, 1999.

[3] R. Yamada, "25-TeV Center of Mass Hadron Collider in VLHC Booster Tunnel," Fermilab-FN-681, May 1999.

[4] "LHC: The Large Hadron Collider", CERN/AC/9505 (LHC), 20 October 1995.

[5] K. Takayama, and J. Kishiro, "Induction synchrotron", NIM A 451, 304, 2000.

[6] K. Takayama, J.Kishiro, M.Sakuda, and M.Wake, "Superbunch Hadron Colliders", submitted to PRL.

[7] E. Nakamura et al.,"Induction Synchrotron: Application to Slow Cycle Synchrotrons", this conference.

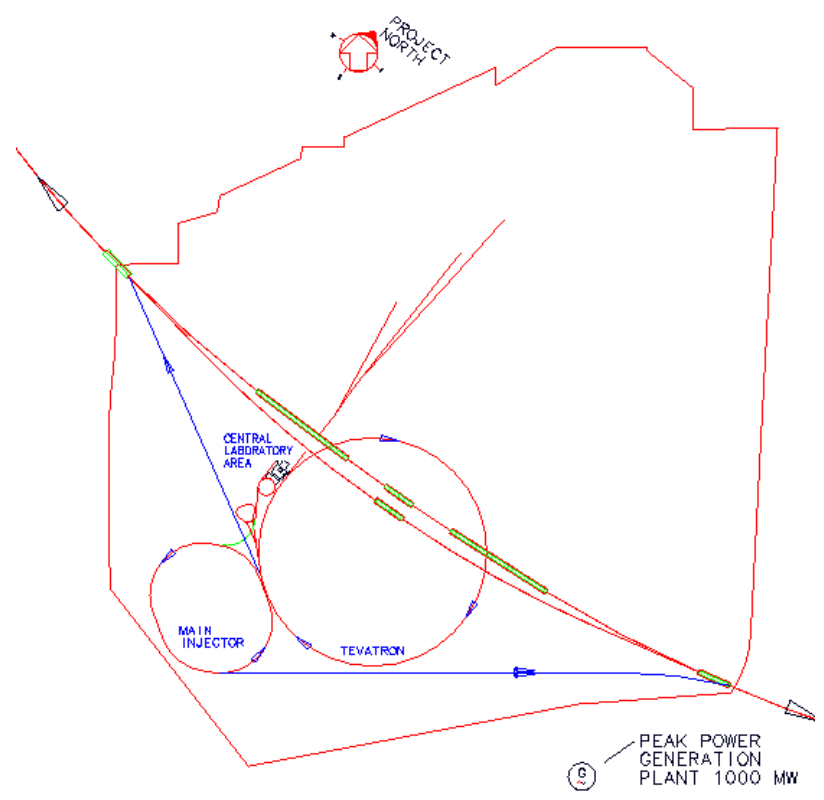

Figure 1. Layout of the tunnels for the low and high field magnet rings inside Fermilab site. The inside tunnel will be used for the 14 and $62 \mathrm{TeV}$ collisions and for their collider detectors. In the second stage the outward bypass is used for the low field magnet ring.

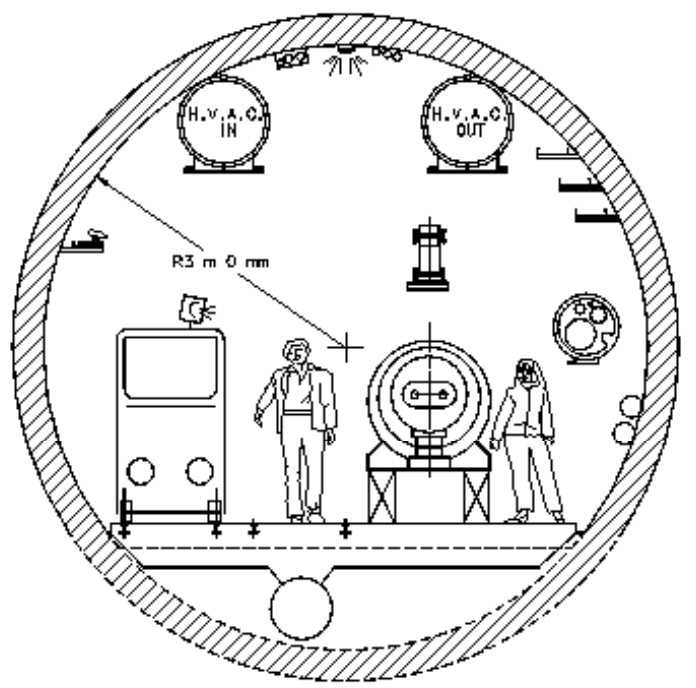

Figure 2. Cross-section of the $62-\mathrm{TeV}$ collider tunnel. It houses the $14-\mathrm{TeV}$ collider magnet and the $62-\mathrm{TeV}$ collider magnet.

Table I. Parameter List of 14-TeV and 62-TeV Colliders.

\begin{tabular}{|l|c|r|r|}
\hline & Unit & \multicolumn{1}{|c|}{$14-\mathrm{TeV}$} & $62-\mathrm{TeV}$ \\
\hline Energy of Ring & $\mathrm{TeV}$ & 7 & 31 \\
\hline Dipole Field & Tesla & 2 & 10 \\
\hline Injection Energy & $\mathrm{TeV}$ & 0.15 & 7 \\
\hline Injection Field & Tesla & 0.0429 & 2.258 \\
\hline Luminosity & $/ \mathrm{cm}^{2} / \mathrm{s}$ & $1.5 \times 10^{35}$ & $5 \times 10^{34}$ \\
\hline Ring Circumference & $\mathrm{km}$ & 87.249 & 87.249 \\
\hline Arc Circumference & $\mathrm{km}$ & 73.304 & 64.926 \\
\hline Dipole Filling Factor & $\%$ & 90 & 80 \\
\hline Dpl Mag. Field Radius & $\mathrm{km}$ & 11.667 & 10.333 \\
\hline No. of P / Bunch & & $1.3 \times 10^{14}$ & $7.8 \times 10^{13}$ \\
\hline No. Bunch / Beam & & 114 & 26 \\
\hline No. of P / Beam & & $1.5 \times 10^{16}$ & $2 \times 10^{15}$ \\
\hline Ave. Beam Current & $\mathrm{A}$ & 8.16 & 1.1 \\
\hline Synchro. Rad. Loss/B & $\mathrm{W} / \mathrm{m}$ & 0.18 & 12 \\
\hline Unit Ind. Cell Lngth & $\mathrm{m}$ & 0.2 & 0.1 \\
\hline Unit Ind. Cell Volt & $\mathrm{kV}$ & 2.5 & 2.5 \\
\hline Rotation Period & $\mu \mathrm{sec}$ & 290.83 & 290.83 \\
\hline Acceleration Energy & $\mathrm{TeV}$ & 6.85 & 24 \\
\hline Acceleration Period & $\mathrm{sec}$ & 1620 & 3620 \\
\hline Accele. Voltage/turn & $\mathrm{kV} / \mathrm{t}$ & 1090.6 & 1938.8 \\
\hline Syn.Rad. Loss /cycle/p & $\mathrm{keV}$ & 1.9 & 940 \\
\hline Total V. for BB-IC & $\mathrm{kV}$ & 93 & 93 \\
\hline Ttl No. of Ac IC/B & $\#$ & 437 & 1152 \\
\hline Ttl Length of Ac IC/B & $\mathrm{m}$ & 87.4 & 115 \\
\hline Coreloss of Unit Cell & $\mathrm{kW}$ & $2.0 \times 2$ & 0.45 \\
\hline Total Coreloss/beam & $\mathrm{kW}$ & 1,748 & 518 \\
\hline SuperBunch Spacing & $\mathrm{m}$ & 765 & 3356 \\
\hline SuperBunch Length & $\mathrm{m}$ & 300 & 150 \\
\hline Time Period /SB & $\mu \mathrm{sec}$ & 2.55 & 11.19 \\
\hline Rep. Rate of IC Pulser & $\mathrm{kHz}$ & 392 & 89.4 \\
\hline
\end{tabular}

